\title{
Comparative study of thyroid function and types of thyroid dysfunction in two areas in Denmark with slightly different iodine status
}

\author{
Nils Knudsen ${ }^{1,2,5}$, Inge Bülow ${ }^{3,5}$, Torben Jørgensen ${ }^{2,5}$, Peter Laurberg ${ }^{3,5}$, Lars Ovesen ${ }^{4,5}$ and Hans Perrild ${ }^{1,5}$ \\ ${ }^{1}$ Department of Internal Medicine I, Bispebjerg Hospital, DK 2400, Copenhagen, Denmark, ${ }^{2}$ Centre for Preventive Medicine, Glostrup Hospital, \\ University of Copenhagen, Copenhagen, Denmark, ${ }^{3}$ Department of Endocrinology, Aalborg Hospital, Denmark, ${ }^{4}$ Institute of Food Research and \\ Nutrition, The Danish Veterinary and Food Administration, Søborg, Denmark and ${ }^{5}$ The Danish Centre for Prevention of Thyroid Diseases (DanThyr), \\ Denmark \\ (Correspondence should be addressed to N Knudsen, Medical Clinic I, Bispebjerg Hospital, DK, 2400 Copenhagen NV, Denmark; Email: \\ danthyr.bbh@dadlnet.dk)
}

\begin{abstract}
Objective: The pattern of thyroid dysfunction seems to depend on the iodine status of the population. Prevalence of thyroid dysfunction could be a parameter to consider when evaluating iodine deficiency disorders in a population.

Design: Comparative cross-sectional investigation in two regions in Denmark with marginally different iodine excretion.

Methods: A random selection of 4649 participants from the Civil Registration System in Denmark in age groups between 18 and 65 years were examined. Thyroid dysfunction was evaluated from blood samples and questionnaires, and compared with results from ultrasonography.

Results: Median iodine excretion was $53 \mu \mathrm{g} / \mathrm{l}$ in Aalborg and $68 \mu \mathrm{g} / \mathrm{l}$ in Copenhagen. Previously diagnosed thyroid dysfunction was found with the same prevalence in the regions. Serum TSH was lower in Aalborg than in Copenhagen $(P=0.003)$ and declined with age in Aalborg, but not in Copenhagen. Not previously diagnosed hyperthyroidism was found with the same overall prevalence in the regions, but in age $>40$ years hyperthyroidism was more prevalent in Aalborg (1.3 vs 0.5\%, $P=0.017)$. Not previously diagnosed hypothyroidism was found more frequently in Aalborg (0.6 vs $0.2 \%, P=0.03)$. Hyperthyroidism was more often associated with macronodular thyroid structure at ultrasound in Aalborg and hypothyroidism was more often associated with patchy thyroid structure in Copenhagen.

Conclusions: Significant differences in thyroid dysfunction were found between the regions with a minor difference in iodine excretion. The findings are in agreement with a higher prevalence of thyroid autonomy among the elderly in the most iodine-deficient region.
\end{abstract}

European Journal of Endocrinology 143 485-491

\section{Introduction}

It has previously been demonstrated that the pattern of thyroid dysfunction is different in areas with clear iodine repletion and moderate iodine deficiency (1). In iodine-replete areas, a higher incidence of sub-clinical and overt hypothyroidism is found, whereas sub-clinical and overt hyperthyroidism is found with a higher incidence in iodine-deficient areas, especially among the elderly. An ecological comparison of previous population-based studies from a recent review (2) does not unambiguously lead to the same conclusion, partly because of methodological differences, partly because most studies were conducted in iodine-replete areas. Recent studies from iodine-deficient $(3,4)$ and mildly iodine-deficient areas (5) confirmed the tendency towards higher prevalences of hypothyroidism in iodine repletion and higher prevalences of hyperthyroidism in iodine deficiency when compared with previous studies.

Hyperthyroidism in iodine deficiency is believed to be associated with thyroid autonomy with a high prevalence of thyroid nodules, whereas autoimmunity is more often the cause of hyperthyroidism - Graves' disease - in iodine-replete areas (6). Consequently, hyperthyroidism has a lower age of onset in iodinesufficient areas, as Graves' disease is seen mostly in the young and middle-aged, whereas toxic nodular goitre is primarily seen among the elderly. The high incidence of hypothyroidism in iodine repletion may be due to an 
increased prevalence of thyroid autoimmunity $(7,8)$ as well as an increased susceptibility to hypothyroidism in cases with thyroid autoimmunity $(1,9,10)$.

With our comparative design, we could avoid bias caused by differences in methodology, different principles of classification, and differences in the composition of the cohort. Thus, we could analyse differences in the prevalences of thyroid disorders attributed to the difference in iodine excretion in the two areas with mild and moderate iodine deficiency. Further, we wanted to compare thyroid dysfunction in the two areas with thyroid structure at ultrasonography.

\section{Materials and methods}

In the period between March 1997 and June 1998, a random sample of the population was examined within the groups: women aged 18-22, 25-30, 40-45 and 60-65 years and men aged 60-65 years. The cohort was drawn from the Civil Registration System in which all subjects living in Denmark are registered, and subjects were included from the north-western part of the municipality of Copenhagen and the central part of Aalborg, a city in the north-western part of Denmark. These regions were chosen as previous studies have demonstrated a difference in iodine excretion between the regions $(5,11)$ probably conditioned by different iodine content of drinking water (12). Otherwise, the regions are comparable as they are both urban areas and no major differences in genetic background should be present. In total, 40233 subjects were included in the sample, and they were all given random numbers within the groups by a PC program and subsequently invited in the order of the random numbers until the desired number of participants in each group was obtained.

The subjects were asked to participate by letter, and in case of no response another letter was sent. If there was still no response or the participants refused to participate in the full examination, they were asked to answer a short questionnaire concerning, among other things, previous thyroid disease and familial occurrence of thyroid disease.

The participants answered questionnaires concerning previous thyroid disease, familial occurrence of thyroid disease and medication. At the investigation, further information was obtained in an interview with an MD, and if there was still uncertainty about previous thyroid disease, medical records were traced.

Blood samples were drawn and stored at $-20{ }^{\circ} \mathrm{C}$, and subsequently the samples were analysed in a sequence ensuring that samples were mixed with respect to region, age, sex and season. Serum thyrotrophin (TSH), free thyroxine $\left(\mathrm{fT}_{4}\right)$ and free tri-iodothyronine $\left(\mathrm{fT}_{3}\right)$ concentrations were analysed with LUMItest (BRAHMS, Berlin, Germany). The functional sensitivity of the TSH assay was $0.01 \mathrm{mU} / \mathrm{l}$. For comparison of sub-clinical thyroid dysfunction between the regions, a reference interval for serum TSH was defined as $0.4-3.6 \mathrm{mU} / \mathrm{l}$, corresponding to the 2.5th and 97.5th percentiles of TSH among participants with no known thyroid disease, TPO Ab $<60 \mathrm{kU} / \mathrm{l}$ (Dynotest RIA, BRAHMS), no thyroid enlargement, and no thyroid nodules at ultrasonography. The reference interval for serum TSH for the detection of overt thyroid dysfunction was set to $0.2-5.0 \mathrm{mU} / \mathrm{l}$, corresponding to the interval used in daily clinical practice and to limits most often used in other epidemiological surveys to allow comparisons. Reference intervals for $\mathrm{fT}_{3}$ and $\mathrm{fT}_{4}$ were defined as 2.5 th to 97.5 th percentiles after exclusion of participants with known thyroid disease or serum TSH outside the reference interval, resulting in a reference interval of 9.8-20.4 pmol/l for $\mathrm{fT}_{4}$ and 3.6-6.9 pmol/l for $\mathrm{fT}_{3}$. Consequently, euthyroidism was defined as a serum TSH concentration in the range $0.4-3.6 \mathrm{mU} / \mathrm{l}$. Sub-clinical hyperthyroidism was defined as a serum $\mathrm{TSH}<0.4 \mathrm{mU} / \mathrm{l}$ and no overt hyperthyroidism defined as TSH $<0.2 \mathrm{mU} /$ $\mathrm{l}$ and $\mathrm{fT}_{3}>6.9 \mathrm{pmol} / \mathrm{l}$ or $\mathrm{TSH}<0.2 \mathrm{mU} / \mathrm{l}$ and $\mathrm{fT}_{4}>$ $20.4 \mathrm{pmol} / \mathrm{l}$. Sub-clinical hypothyroidism was defined as serum TSH $>3.6 \mathrm{mU} / \mathrm{l}$ and no overt hypothyroidism defined as serum TSH $>5 \mathrm{mU} / \mathrm{l}$ and $\mathrm{fT} 4<9.8 \mathrm{pmol} / \mathrm{l}$.

Thyroid ultrasonography was performed with a Siemens Sonoline Versa Pro with a $7.5 \mathrm{MHz} 70 \mathrm{~mm}$ linear transducer, effective length $62 \mathrm{~mm}$ (Siemens, Erlangen, Germany). Thyroid volume was calculated as length $\times$ width $\times$ depth $\times \pi / 6$ for each lobe. Thyroid nodules exceeding $5 \mathrm{~mm}$ in diameter were registered; in cases of more than three nodules in a lobe, only the three largest were registered. The thyroid echo pattern was classified as diffuse or patchy. An evaluation of the inter-observer variation of these variables showed good agreement for thyroid volume and number of nodules, but little agreement for echo pattern (13). Echo pattern was consequently only used for comparisons within each region.

Casual urine samples were collected during the time interval 0800 to $1900 \mathrm{~h}$ and analysed for iodine concentration by the Ce/As method after digestion by alkaline ashing as previously described $(14,15)$. The median iodine excretion was $53 \mu \mathrm{g} / \mathrm{l}(0.42 \mu \mathrm{mol} / \mathrm{l})$ in Aalborg and $68 \mu \mathrm{g} / \mathrm{l}(0.54 \mu \mathrm{mol} / \mathrm{l})$ in Copenhagen (Fig. 1); after exclusion of subjects taking individual iodine supplementation the figures were $45 \mu \mathrm{g} / \mathrm{l}$ $(0.35 \mu \mathrm{mol} / \mathrm{l})$ and $61 \mu \mathrm{g} / \mathrm{l}(0.48 \mu \mathrm{mol} / \mathrm{l})$ respectively. According to WHO criteria (16), the Aalborg region can be classified as moderately iodine-deficient and the Copenhagen region can be classified as mildly iodinedeficient.

The study was approved by the regional Ethics Committee in Copenhagen and Northern Jutland and all participants gave written informed consent.

\section{Statistics}

Data processing was done with SPSS version 8.0 software. Non-parametric statistics were used for the analysis of continuous data (Mann-Whitney and 


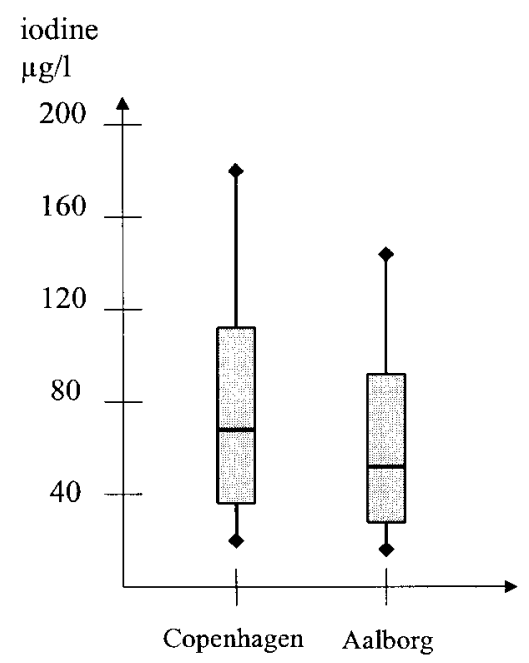

Figure 1 The distribution of iodine concentrations in spot urine samples from subjects in two regions in Denmark. Urine samples were available from 4616 participants (99.3\%). Boxes represent median and inter-quartile range; whiskers represent 10th and 90th percentiles. $P$ for regional difference $<0.001$ (Mann-Whitney).

Kruskal-Wallis), and general linear models were used to analyse the relation between a dependent and more than one independent variable; logarithmic transformation was then used for continuous variables. For the TSH analyses data were adjusted for the sampling hour, as the age and sex groups were not equally distributed throughout the day and serum TSH varied through the day. For dichotomous variables Pearson Chi square tests and logistic regression analysis were used. An interaction parameter was included in the logistic regression analyses to test for differences in the relative distribution of a variable between the regions. The level of significance was set to $5 \%$.

\section{Results}

\section{Participation}

The participation rate was $50.1 \%$, as 4649 subjects participated out of the 9274 invited. The short questionnaire was answered by another $26.8 \%$ and $23.1 \%$ did not respond. The participation rate was higher in Aalborg than in Copenhagen ( 54.6 vs $46.6 \%$, $P<0.001)$, and the short questionnaire was answered by the same fraction of the cohort in the two regions ( $26.5 \mathrm{vs} 27.0 \%$ ). Results from the thyroid function tests were available on 4583 subjects $(98.6 \%)$ and results from the ultrasound investigation were available on 4642 subjects $(99.8 \%)$.

In a comparison of self-reported thyroid dysfunction among participants and responders to the short questionnaire only (non-participants), no significant differences were found between participants and non-participants for hyperthyroidism (participants $2.4 \%$, non-participants $1.8 \%$, non significant) or hypothyroidism (participants 2.9\%, non-participants $2.8 \%$, non significant), whereas bias was found regarding self-reported goitre $(5.9 \%$ vs $3.6 \%, P<0.001)$. Familial occurrence of thyroid dysfunction was also reported more often by participants $(8.0 \%)$ than nonparticipants $(4.8 \%), P<0.001$; however, only minor difference in the prevalence of thyroid dysfunction in this study was found between participants with or without familial occurrence of thyroid disease $(1.4 \%$ vs $1.1 \%)$. No differences in the degree of selection bias were found between the regions.

\section{Previously diagnosed thyroid dysfunction}

To the question, 'has a doctor ever told you that you had hyperthyroidism?', 133 answered yes, but after the interview and tracing of medical records, the number of participants with previous hyperthyroidism was 101 (92 cases confirmed and nine additional cases identified), yielding a prevalence of previously diagnosed hyperthyroidism of $2.2 \%$. To the corresponding question concerning hypothyroidism, positive answers were given in 109 cases. After the interview only 53 cases were confirmed and another seven cases were identified, yielding a prevalence of previously diagnosed hypothyroidism of $1.3 \%$.

The prevalence of previously diagnosed hyperthyroidism was $1.9 \%$ in mild iodine deficiency (ID) and $2.5 \%$ in moderate ID, and the prevalence of hypothyroidism was $1.2 \%$ in mild ID and $1.4 \%$ in moderate ID. The prevalence of previously diagnosed thyroid dysfunction was obviously increasing with age, but no significant regional differences were found overall or in any of the age groups. Two cases of thyroiditis and two cases of thyroid cancer had been diagnosed in each region.

\section{Thyroid function tests}

Serum TSH concentrations showed significant regional differences caused by a decline in TSH with age in the moderate ID area, a decline that was not present in the mild ID area (Table 1). A significant regional difference in TSH among the elderly men also contributed to the overall regional difference in TSH. If an adjustment for the nodularity of the thyroid was introduced in the analysis, the regional differences in TSH were only seen in subjects with nodular glands, whereas subjects without nodules had similar TSH levels in the two regions $(P=0.002$ for interaction in a linear model $)$. No significant overall regional difference was found for serum $\mathrm{fT}_{3}$ or serum $\mathrm{fT}_{4}$. A parallel decline from 5.28 to $5.10 \mathrm{pmol} / \mathrm{l}$ (median) in free $\mathrm{T}_{3}(P<0.001$, linear model) and increase in free $\mathrm{T}_{4}$ from 13.9 to $15.2 \mathrm{pmol} / \mathrm{l}$ $(P<0.001)$ with age among women was found in the two regions (data not shown). 
Table 1 Mean serum TSH after logarithmic transformation in 4356 participants never treated for thyroid disease from two regions in Denmark with mild (Copenhagen) and moderate (Aalborg) iodine deficiency. Serum TSH values correspond to morning values. $P$ values for the regional difference and the difference between age groups in linear models are given. Values in parentheses are $95 \%$ confidence intervals for the mean.

\begin{tabular}{lccc}
\hline Sex and age & $\begin{array}{c}\text { TSH mild ID } \\
\mathrm{mU} / \mathrm{I}(95 \% \mathrm{Cl})\end{array}$ & $\begin{array}{c}\text { TSH moderate ID } \\
\mathrm{mU} / \mathrm{l}(95 \% \mathrm{Cl})\end{array}$ & $\begin{array}{c}\boldsymbol{P} \text { (between } \\
\text { regions) }\end{array}$ \\
\hline Women, 18-22 & $1.44(1.35-1.55)$ & $1.49(1.38-1.60)$ & 0.62 \\
Women, 25-30 & $1.49(1.39-1.60)$ & $1.40(1.30-1.52)$ & 0.26 \\
Women, 40-45 & $1.39(1.29-1.50)$ & $1.27(1.17-1.37)$ & 0.05 \\
Women, 60-65 & $1.31(1.21-1.42)$ & $1.14(1.05-1.25)$ & 0.004 \\
Men, 60-65 & $1.36(1.27-1.47)$ & $1.23(1.13-1.43)$ & 0.04 \\
Total & $1.40(1.33-1.47)$ & $1.31(1.25-1.37)$ & 0.004 \\
$P$ (between groups) & 0.15 & $<0.001$ & \\
\hline
\end{tabular}

\section{Not previously diagnosed thyroid dysfunction}

The prevalence of thyroid dysfunction among participants who had never been treated for thyroid disease is illustrated in Table 2. The prevalence of hyperthyroidism as well as hypothyroidism including subclinical disease was increasing with age $(P<0.001$, Pearson Chi-square) and the prevalence was higher among women than among age-matched men $(P<0.001)$. Not previously diagnosed hyperthyroidism was found with the same prevalence in the two regions when all age groups were included. The tendency was that hyperthyroidism was diagnosed more often in the younger age groups in mild ID $(P=0.057)$, and conversely a significantly higher prevalence of not previously diagnosed hyperthyroidism was found in moderate ID among participants aged more than 40 years $(P=0.017)$. Combining the cases with previous and present thyroid dysfunction did not yield further statistically significant regional differences. When all age groups were included, a small difference in the prevalence of sub-clinical hyperthyroidism was found $(P=0.049)$. When only subjects over the age of 40 years were included, the regional difference in sub-clinical hyperthyroidism was more pronounced $(7.8 \%$ in moderate ID vs $5.3 \%$ in mild ID, $P=0.01$ ).

The prevalence of overt hypothyroidism was higher in moderate ID than in mild ID $(P=0.03)$ (Table 2). Though a tendency was seen towards a higher prevalence of sub-clinical hypothyroidism in mild ID than in moderate ID in subjects aged 40 years or more ( 5.4 vs $4.0 \%, P=0.097$ ), no significant differences in the prevalence of sub-clinical hypothyroidism were found.

For comparison with other studies, sub-clinical hyperthyroidism was also computed as TSH $<0.2 \mathrm{mU} /$ $\mathrm{l}$, which was found in $1.8 \%$ in mild ID and $2.0 \%$ in moderate ID $(P=0.64)$; and sub-clinical hypothyroidism was defined as TSH $>5 \mathrm{mU} / \mathrm{l}$, which was found in $2.0 \%$ in mild ID and $1.8 \%$ in moderate ID $(P=0.69)$.

\section{Thyroid dysfunction and thyroid structure}

Thyroid structure at ultrasound in different groups of thyroid function is demonstrated in Fig. 2. Macronodular thyroid structure was found in $75.9 \%$ of participants with suppressed TSH $(<0.2 \mathrm{mU} / \mathrm{l})$ and in $27.8 \%$ of participants with normal TSH (OR 3.7; CI 2.0-6.8 in a logistic regression analysis adjusting for the possible confounding by age). Correspondingly, thyroid enlargement defined as a thyroid volume exceeding $18 \mathrm{ml}$ for women and $25 \mathrm{ml}$ for men (17) was found in $63 \%$ of participants with suppressed TSH, in $17 \%$ of participants with normal TSH, and in $10 \%$ of participants with elevated TSH.

Likewise, patchy thyroid structure at ultrasound was found in $51.8 \%$ of participants with elevated TSH and in $13.5 \%$ participants with normal TSH (OR 6.2; CI 4.0-9.8 in logistic regression analysis adjusting for age and sex).

\section{Discussion}

In this study, comparisons in a Danish cohort are made between two regions that differ slightly with respect to iodine excretion but that are otherwise comparable. This regional difference in iodine excretion has been relatively constant since the first major investigation more than 30 years ago (11). No differences were found in the prevalence of previously diagnosed thyroid dysfunction in any age group, but some differences in the prevalence of not previously diagnosed thyroid dysfunction were found.

Serum TSH levels differed significantly between the regions. Lower levels of TSH were found in the moderate ID area than in the mild ID area due to a decline in TSH with age in moderate ID, a decline not observed in mild ID. In an iodine-sufficient area in Sweden, TSH increased with age, though the difference was not significant (18), and in an iodine-deficient area in Italy, an age-related decline in TSH has been reported (4). In a 
Table 2 Thyroid function in 4360 participants never treated for thyroid disease in two areas in Denmark with mild (Copenhagen) or moderate (Aalborg) iodine deficiency.

\begin{tabular}{|c|c|c|c|c|c|c|c|c|c|c|c|c|}
\hline \multirow[b]{2}{*}{ Sex and age } & \multicolumn{2}{|c|}{ Hyperthyroid } & \multicolumn{2}{|c|}{$\begin{array}{l}\text { Sub-clinical } \\
\text { hyperthyroid }\end{array}$} & \multicolumn{2}{|c|}{ Euthyroid } & \multicolumn{2}{|c|}{$\begin{array}{l}\text { Sub-clinical } \\
\text { hypothyroid }\end{array}$} & \multicolumn{2}{|c|}{ Hypothyroid } & \multicolumn{2}{|c|}{ Total } \\
\hline & $n$ & $\%$ & $n$ & $\%$ & $n$ & $\%$ & $n$ & $\%$ & $n$ & $\%$ & $n$ & $\%$ \\
\hline \multicolumn{13}{|l|}{ Mild ID } \\
\hline Women, 18-22 & 1 & 0.2 & 13 & 2.7 & 459 & 94.6 & 12 & 2.5 & 0 & & 485 & 100.0 \\
\hline Women, 25-30 & 3 & 0.6 & 11 & 2.3 & 447 & 92.2 & 22 & 4.5 & 2 & 0.4 & 485 & 100.0 \\
\hline Women, 40-45 & 2 & 0.4 & 17 & 3.7 & 421 & 90.9 & 22 & 4.8 & 1 & 0.2 & 463 & 100.0 \\
\hline Women, 60-65 & 4 & 1.0 & 28 & 7.3 & 319 & 82.6 & 34 & 8.8 & 1 & 0.3 & 386 & 100.0 \\
\hline Men, 60-65 & 0 & & 19 & 4.0 & 442 & 93.3 & 13 & 2.7 & 0 & & 474 & 100.0 \\
\hline Total & 10 & 0.4 & 88 & 3.8 & 2088 & 91.1 & 103 & 4.5 & 4 & 0.2 & 2293 & 100.0 \\
\hline \multicolumn{13}{|l|}{ Moderate ID } \\
\hline Women, 18-22 & 0 & & 11 & 2.5 & 412 & 93.0 & 16 & 3.6 & 4 & 0.9 & 443 & 100.0 \\
\hline Women, 25-30 & 0 & & 11 & 2.5 & 410 & 94.2 & 12 & 2.8 & 2 & 0.5 & 435 & 100.0 \\
\hline Women, 40-45 & 3 & 0.7 & 20 & 5.0 & 372 & 92.1 & 9 & 2.2 & 0 & & 404 & 100.0 \\
\hline Women, 60-65 & 11 & 3.1 & 28 & 8.0 & 284 & 80.8 & 25 & 7.1 & 4 & 1.1 & 352 & 100.0 \\
\hline Men, 60-65 & 2 & 0.5 & 29 & 6.7 & 393 & 90.7 & 7 & 1.6 & 2 & 0.5 & 433 & 100.0 \\
\hline Total & 16 & 0.8 & 99 & 4.8 & 1871 & 90.5 & 69 & 3.3 & 12 & 0.6 & 2067 & 100.0 \\
\hline
\end{tabular}

Regional differences in total prevalences were analysed with Chi-square tests: $P=0.15$ for hyperthyroidism; $P=0.049$ for sub-clinical hyperthyroidism; $P=0.21$ for sub-clinical hypothyroidism; $P=0.03$ for hypothyroidism.

comparative study, higher median TSH was reported in an iodine-replete area than in an iodine-deficient area (19). Thus, in moderate iodine deficiency, a reduced serum TSH is observed compared with serum TSH in areas with a lesser degree of iodine deficiency or iodine sufficiency. These reduced TSH levels especially among the elderly are in contrast to increased TSH as a direct

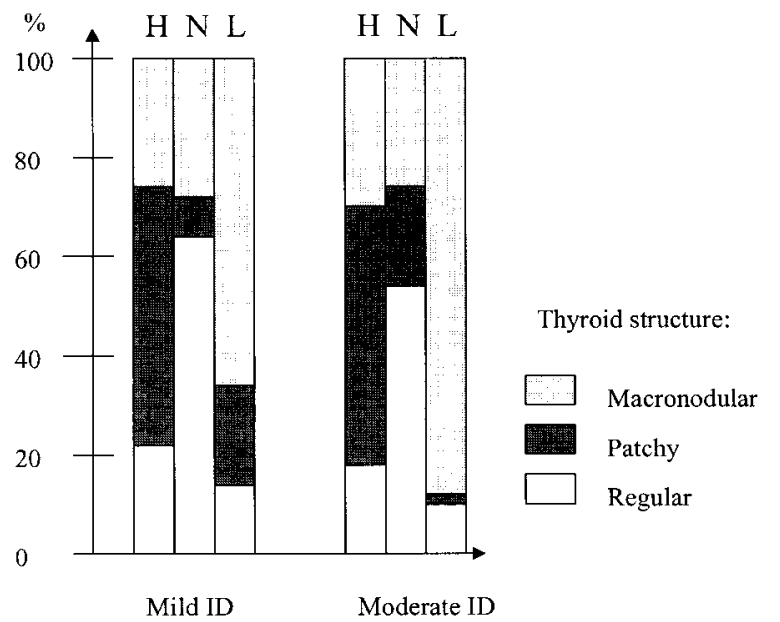

Figure 2 Thyroid structure stratified by TSH level and region in 4357 subjects from two areas in Denmark with mild (Copenhagen) and moderate (Aalborg) iodine deficiency. Subjects with known thyroid disease were excluded. $\mathrm{H}=\mathrm{High} \mathrm{TSH}(>5 \mathrm{mU} / \mathrm{l}), \mathrm{N}=$ Normal TSH $(0.2-5 \mathrm{mU} / \mathrm{l}), \mathrm{L}=\mathrm{Low} \mathrm{TSH}(<0.2 \mathrm{mU} / \mathrm{l})$. Interaction terms in a logistic regression analysis were used to describe regional differences; suppressed TSH was more closely associated with macronodular thyroid structure in moderate ID than in mild ID $(P=0.02)$, and elevated TSH was more closely related to patchy thyroid structure in mild ID than in moderate ID $(P=0.02)$. effect of iodine deficiency as observed in severe iodine deficiency (20). This is presumably due to autonomous functioning thyroid tissue, as this regional difference in serum TSH was only found in subjects with nodular glands, and it is in accordance with data from moderate iodine deficiency in Italy, where decreasing levels of serum TSH were found with increasing degrees of goitre at a clinical examination (21).

A decline in serum TSH with age corresponds to the finding of an increased prevalence of overt as well as sub-clinical hyperthyroidism among the elderly in moderate ID. Likewise, not previously diagnosed overt hypothyroidism was more prevalent in moderate ID than in mild ID; it should be emphasized, however, that this difference is based on few cases. The major difference in thyroid dysfunction between iodinedeficient and iodine-replete areas has been reported in the older age groups (6). This is in accordance with an increasing prevalence of toxic nodular goitre with age in this study, especially in moderate ID. Consequently, if older age groups had been investigated, regional differences might have been more pronounced. That is, however, not possible in a set-up like ours, as participation rates would be too low and selection of the healthiest would occur to a large extent.

Our data indicate that, apart from a reduction in nontoxic goitre, a reduction in hyperthyroidism may be expected in the long run among the oldest part of the population following an increase in the iodine intake of a population as reported from other countries, though a temporary increase in the incidence of thyrotoxicosis has been reported from most studies after the initiation of iodization programmes (22-24). 
Comparisons of prevalences with previous studies is problematic due to the composition of this cohort with respect to age and sex and differences in the definitions of thyroid dysfunction. By selecting the relevant strata and paying attention to different cut-off levels for TSH, some comparisons could be made. Elevation of serum TSH was less common in our study than reported in most studies from iodine-replete areas in a recent review (2), and more common than in an iodine-deficient area (3). Suppressed TSH, on the other hand, seems less common than in iodine-deficient areas and more common than in iodinereplete areas as discussed in a previous paper (5).

When previously diagnosed thyroid disease is compared, the registration procedure needs to be accounted for. We found a considerable over-reporting of thyroid dysfunction in the questionnaires compared with data obtained from interviews and tracing of records, though only cases diagnosed by a physician were asked for in the questionnaire. Hyperthyroidism was over-reported by $32 \%$ and hypothyroidism by $82 \%$, probably because weight problems are often attributed to thyroid dysfunction. Tunbridge et al. described a similar over-reporting of hyperthyroidism and a smaller over-reporting of hypothyroidism (25).

The participation rate in the full investigation was only $50 \%$ in this study, but no selection bias seemed to be present. Though bias might be caused by an over-representation of subjects with familial occurrence of thyroid dysfunction or with goitre among the participants, it was of little importance, as especially familial occurrence of thyroid disease was not a strong predictor of thyroid dysfunction, and as self-reported thyroid dysfunction was equally present among participants and responders to a short questionnaire only. Further, it should be emphasized that comparisons between the regions seemed unbiased, as no signs of difference in selection bias between the regions were found.

In conclusion, hyperthyroidism has a different age of onset in the two regions. A higher prevalence of not previously diagnosed hyperthyroidism was found in the area with the lowest iodine excretion due to an increased prevalence of hyperthyroidism in the oldest age groups. Corresponding to this, a decline in serum TSH and increased nodularity among participants with suppressed TSH was found in the region with the lowest iodine excretion, supporting the theory of increased autonomy in iodine-deficient areas.

\section{Acknowledgements}

This study was supported by grants from the Tømmerhandler Vilhelm Bang Foundation, the Copenhagen Hospital Corporation Research Foundation, the 1991 Pharmacy Foundation, the Danish Medical Foundation, the Health Insurance Foundation, North Jutland County Research Foundation and BRAHMS Diagnostica. We also express our thanks to Ingelise Legaard and Rene Fiege who carefully performed the ultrasonography and Erik Christiansen who assisted with the iodine analyses.

\section{References}

1 Laurberg P, Pedersen KM, Hreidarsson A, Sigfusson N, Iversen E \& Knudsen PR. Iodine intake and the pattern of thyroid disorders: a comparative epidemiological study of thyroid abnormalities in the elderly in Iceland and in Jutland, Denmark. Journal of Clinical Endocrinology and Metabolism 199883 765-769.

2 Wang C \& Crapo LM. The epidemiology of thyroid disease and implications for screening. Endocrinology and Metabolism Clinics of North America 199726 189-218.

3 Seck T, Scheidt NC, Ziegler R \& Pfeilschifter J. Pravalenz von Schilddrusenfunktionsstorungen bei 50 - bis 80 jahrigen. Eine epidemiologische Querschnittsstudie in einer sudwestdeutschen Gemeinde. Medizinische Klinik 199792 642-646.

4 Aghini-Lombardi F, Antonangeli L, Martino E, Vitti P, Maccherini $\mathrm{D}$, Leoli $\mathrm{F}$ et al. The spectrum of thyroid disorders in an iodinedeficient community: the Pescopagano survey. Journal of Clinical Endocrinology and Metabolism 199984 561-566.

5 Knudsen N, Jørgensen T, Rasmussen S, Christiansen E \& Perrild H. The prevalence of thyroid dysfunction in a population with borderline iodine deficiency. Clinical Endocrinology $199951361-$ 367.

6 Laurberg P, Pedersen KM, Vestergaard H \& Sigurdsson G. High incidence of multinodular toxic goitre in the elderly population in a low iodine intake area vs. high incidence of Graves' disease in the young in a high iodine intake area: comparative surveys of thyrotoxicosis epidemiology in East-Jutland Denmark and Iceland. Journal of Internal Medicine 1991229 415-420.

7 Kahaly GJ, Dienes HP, Beyer J \& Hommel G. Iodide induces thyroid autoimmunity in patients with endemic goitre: a randomised, double-blind, placebo-controlled trial. European Journal of Endocrinology $1998139290-297$.

8 Tsatsoulis A, Johnson EO, Andricula M, Kalogera C, Svarna E, Spyroy $\mathrm{P}$ et al. Thyroid autoimmunity is associated with higher urinary iodine concentrations in an iodine-deficient area of Northwestern Greece. Thyroid 19999 279-283.

9 Szabolcs I, Podoba J, Feldkamp J, Dohan O, Farkas I, Sajgo M et al. Comparative screening for thyroid disorders in old age in areas of iodine deficiency, long-term iodine prophylaxis and abundant iodine intake. Clinical Endocrinology 199747 87-92.

10 Sundick RS, Bagchi N \& Brown TR. The role of iodine in thyroid autoimmunity: from chickens to humans: a review. Autoimmunity $19921361-68$

11 Pedersen KM, Nohr SB \& Laurberg P. Jodindtagelse i Danmark. Ugeskrift for Laeger 1997159 2201-2206.

12 Pedersen KM, Laurberg P, Nohr S, Jorgensen A \& Andersen S. Iodine in drinking water varies by more than 100-fold in Denmark. Importance for iodine content of infant formulas. European Journal of Endocrinology 1999140 400-403.

13 Knudsen N, Bols B, Bulow I, Jorgensen T, Perrild H, Ovesen L et al. Validation of ultrasonography of the thyroid gland for epidemiological purposes. Thyroid 19999 1069-1074.

14 Wilson B \& Van Zyl A. The estimation of iodine in thyroidal amino acids by alkaline ashing. South African Journal of Medical Science $19673270-82$.

15 Laurberg P. Thyroxine and 3, 5, 3'-triiodothyronine content of thyroglobulin in thyroid needle aspirates in hyperthyroidism and hypothyroidism. Journal of Clinical Endocrinology and Metabolism $198764969-974$.

16 Delange F. The disorders induced by iodine deficiency. Thyroid 19944 107-128.

17 Gutekunst R, Becker W, Hehrmann R, Olbricht T \& Pfannenstiel P. Ultraschalldiagnostik der Schilddruse. Deutsche Medinzinische Wochenschrift 1988113 1109-1112.

18 Petersen K, Lindstedt G, Lundberg PA, Bengtsson C, Lapidus L \& Nystrom E. Thyroid disease in middle-aged and elderly Swedish 
women: thyroid-related hormones, thyroid dysfunction and goitre in relation to age and smoking. Journal of Internal Medicine 1991 229 407-413.

19 Gutekunst R, Smolarek H, Hasenpusch U, Stubbe P, Friedrich HJ, Wood WG et al. Goitre epidemiology: thyroid volume, iodine excretion, thyroglobulin and thyrotropin in Germany and Sweden. Acta Endocrinologica 1986112 494-501.

20 Chopra IJ, Hershman JM \& Hornabrook RW. Serum thyroid hormone and thyrotropin levels in subjects from endemic goiter regions of New Guinea. Journal of Clinical Endocrinology and Metabolism $197540326-333$.

21 Roti E, Gardini E, D’Amato L, Salvi M, Robuschi G, Manfredi A et al. Goiter size and thyroid function in an endemic goiter area in northern Italy. Journal of Clinical Endocrinology and Metabolism $198663558-563$.

22 Baltisberger BL, Minder CE \& Burgi H. Decrease of incidence of toxic nodular goitre in a region of Switzerland after full correction of mild iodine deficiency [see comments]. European Journal of Endocrinology 1995132 546-549.

23 Mostbeck A, Galvan G, Bauer P, Eber O, Atefie K, Dam K et al. The incidence of hyperthyroidism in Austria from 1987 to 1995 before and after an increase in salt iodization in 1990. European Journal of Nuclear Medicine 199825 367-374.

24 Garcia-Mayor RV, Rios M, Fluiters E, Mendez LF, Garcia-Mayor EG

\& Andrade A. Effect of iodine supplementation on a pediatric population with mild iodine deficiency. Thyroid $199991089-$ 1093.

25 Tunbridge WM, Evered DC, Hall R, Appleton D, Brewis M, Clark F et al. The spectrum of thyroid disease in a community: the Whickham survey. Clinical Endocrinology 19777 481-493.

Received 15 May 2000

Accepted 7 July 2000 